\title{
Hand Vein Authentication System based on Information Sets
}

\author{
Dittu Parashar \\ Jamia Hamdard \\ New Delhi-110062
}

\author{
Ranjit Biswas \\ Jamia Hamdard \\ New Delhi-110062
}

\author{
Jyotsana Grover \\ Jamia Hamdard \\ New Delhi-110062
}

\begin{abstract}
Biometrics have been used from ages for authentication purpose but the key advantages of hand vein pattern that is "hand vein pattern are 100 times more unique than other physiological features" are attracting many researchers. In this paper we have proposed an efficient authentication system using hand vein pattern. The concept of information sets has been used for feature extraction so as to have control over the local information. The information set based features has already been implemented on biometrics like ear and face. Till now these features have not been implemented on hand vein pattern based verification systems hence we have explored their suitability on hand veins. All the experiments have been carried out on hand vein database developed at IIT, DELHI .We have used Hanman classifier to check the accuracy of extracted features. Then we have optimized the Hanman classifier performance by using genetic algorithm. The optimized results shows that the proposed system in $99 \%$ efficient. We have also compared Hanman classifier accuracy with accuracy obtained from knearest neighbor and SVM classifier.
\end{abstract}

\section{Keywords}

Hand vein, Biometrics, Information sets, Fuzzy sets, Hanman classifier, T-norms, Genetic algorithm.

\section{INTRODUCTION}

In this fast growing world, security is the major concern in cyberspace. Due to the rise in technology the crime ratio is increasing day by day. Hence, Privacy protection is the major challenging task in the field of security. To overcome these privacy risks to certain extent some verification methods came into existence. These verification methods are classified into two categories i.e. traditional verification methods and modern verification methods. The traditional verification methods include passwords, PINs, smartcards. The main disadvantage of these systems is that they offer limited security and they are not much reliable. Hence to overcome these drawbacks modern verification methods came into existence. Modern verification methods involve analysis of human physical, biological and behavioral characteristics and were further named as biometrics. Biometrics uses physiological or behavioral characteristics to determine or verify the identity of individual. The physiological characteristics include thumbprint, palm print, face, thermal image, iris pattern, vein pattern etc whereas behavioral characteristics include voice pattern, handwriting, or acoustic signature. The modern verification methods have a big advantage i.e. they are difficult for attacker to copy or forge.

Recently hand vein pattern is attracting the attention of many researchers working in the field of security. Shape of vein patterns in the back of the hand varies from person to person. The key advantages of hand vein pattern over others physiological features are uniqueness, stability and immunity.

a. Uniqueness: Shape of vein patterns in the back of the hand varies from person to person.

b. Stability: Hand vein pattern is stable that is the shape of the vein remains unchanged even when human being grows.

c. Immunity: Hand vein patterns are difficult for attacker to copy and forge.

Hence, these advantages makes it a more consistent biometric for personal identification. Further, the state of skin, temperature and humidity has little effect on the vein image, unlike fingerprint and facial feature acquirement [1]. A lot of researches had been carried out on hand vein patterns and most of the researches are motivating researchers to find a best methods and techniques to develop an efficient hand vein authentication system.

\subsection{Motivation And Related Work}

Various key advantages of hand vein patterns are motivating many researchers to build an efficient authentication system using hand veins. The hand vein authentication system proposed in this paper makes use of the concept of information sets. The principle motive of using the concept of information sets is to limit the uncertainty from gray level values. This concept has been implemented at the stage of feature extraction. In this two types of features has been extracted i.e. energy feature and sigmoid feature. To check the accuracy of these extracted features Hanman classifier has been applied. The motive behind using the hanman classifier is that the computational requirements of this classifier is relatively less as compared to other classifiers and this classifier offers better performance as that of other classifiers. To prove this a comparison has been done to compare the accuracy of hanman classifier with other classifiers. Then we have optimized the hanman classifier by using genetic algorithm to improve its performance to get the best results for the system.

\section{LITERATURE SURVEY}

Recently hand veins patterns are attracting many researchers. Researchers are using different techniques to build an efficient authentication system. Tanaka and kubu [2] developed a hand vein acquisition device using near IR imaging. The verification of the developed system was done by using fast Fourier transform based phase correlation scheme. Further the developed system was tested with noise reduction filters, sharpness filters, histogram manipulation. Wang and leedham [3] have proposed another method for personal authentication. In their work hand vein images were acquired from thermal imaging. The classification was done by using Hausdorff distance and the matching scores were computed between extracted line patterns. Shalin et al. [4] 
proposed an authentication system using fast correlation of near infrared hand vein patterns. They used spatial correlation of hand vein pattern for recognition. In their work after segmentation \&pattern post processing correlation is used to measure identity. Kumar and prathyusha [5] presented a new approach for Personal authentication. Their proposed method was based on hand vein triangulation and knuckle shape. In their proposed system user authentication was done by triangulation of hand vein image and knuckle shape of information palm dorsa hand vein image. In their work ROI was extracted by using knuckle tips. Matching scores were generated based on four topologies of triangulation of binarized vein structured and geometric features consisting of knuckle point perimeter distance in acquired images. The weighted score level combination of these two matching scores were used to authenticate the individual. Wang et al.[6] proposed local scale invariant feature transform (SIFT) which has practical significance due to its translation and rotation invariance. The hand vein image is preprocessed to remove the background and reduce image noises and the SIFT features are extracted to describe the gradient information of hand vein. Minimum distance classifier is used for matching. Wanget al. [7] presented another approach of hand vein recognition system .Their approach was based on partitioning local binary pattern and further was assessed using a similarity measure which was obtained by calculating chi square statistic between the feature vectors of tested sample and the target sample. Wang et al. [8] proposed another approach for recognition which was based on partitioning of local binary pattern (LBP).In their work after preprocessing the image is divided into subimages.LBP features are extracted from all sub images. For classification minimum distance classifier is used. Wang and Liao [9] presented another approach for recognition which is based on feature coding. In their approach the output of partition LBP is extracted and is given as input to back propagation encoder. The orthogonal gold code is selected as output code for back propagation. For final classification correlation classifier is used. Tang et al. [10] presented Hand-dorsa vein recognition based on multi-level key point detection and SIFT feature based local feature matching. In multi-level key point detection approach Harris-Laplace and Hessian-Laplace detectors are combined to localize key points that highlight the more discriminating information. Then SIFT based local matching associates these key points between hand dorsa of the same individual. Huang et al. [11] proposed another approach on hand vein recognition based on oriented gradient maps and local feature matching. The proposed approach make use of oriented gradient maps (OGMs) to represent the hand vein images. Further, SIFT based local matching is performed to associate the key points between corresponding OGM pairs of the same subject.

\section{GENERAL MODEL FOR HAND VEIN AUTHENTICATION SYSTEM}

An authentication system using hand vein pattern includes two modules:

\subsection{User module}

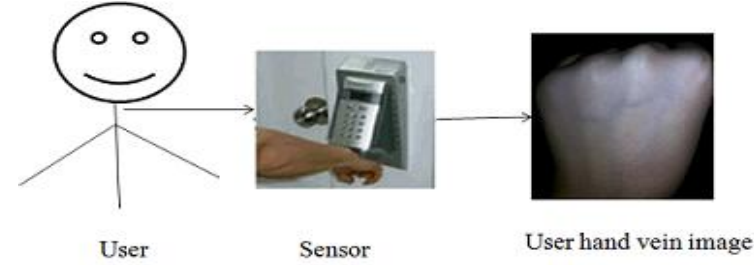

3.2 Authentication server module

It is divided into two steps:

\subsubsection{Preprocessing}

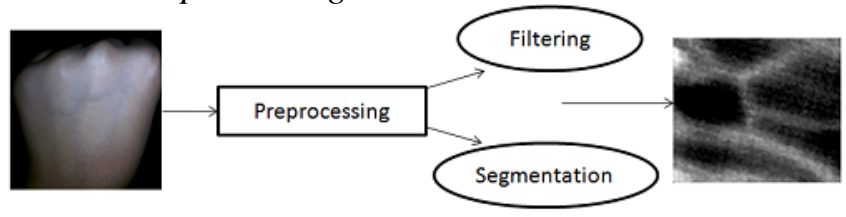

Acquired Image

Preprocessing

ROI Image

3.2.2 Verification

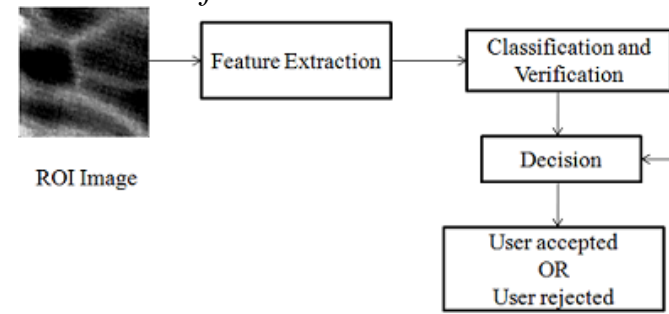

\section{FEATURE EXTRACTION BY USING INFORMATION SETS}

In this paper the concept of information sets has been used for feature extraction. The motive behind using the information concept is to reduce the uncertainty present in gray level values. Information set is defined as a pair of information source and membership function. This information set results in formation of feature vector. The concept of information sets overcomes the limitation of fuzzy set theory by expanding the scope of fuzzy sets. The biggest limitation in fuzzy set theory is that in most of the applications that involve concept of fuzzy set only the membership function is the key to fuzzy logic operations. The value of information source is rarely figured out in fuzzy set theory. Hence this limitation is solved to certain extent by using concept of information sets. In this context we are concerned with limiting the uncertainty from gray level values.

\subsection{Concept Of Information Sets $(H)$}

Information set is a pair of information source values and membership function. This information set results in formation of feature vector. In this context we are concerned with finding the values of information source and membership function. It is noted that 


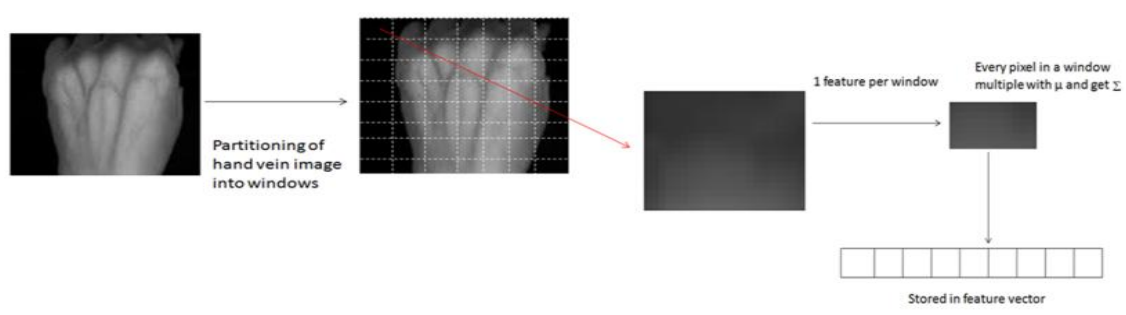

Figure 1: Extraction of Feature Vector from Hand Vein Image

membership function can be exponential, trapezoidal, triangular or Gaussian memberships [12].

Let $I$ be any image. The information source value is denoted by $\left\{\mathrm{I}_{\mathrm{ij}}\right\}$ and is computed by partitioning the image into windows of size $\mathrm{n} X \mathrm{n}$ as shown in Fig.1. Now consider the fuzzy set which is a pair of gray level values and their membership function values. Gray level values are denoted by $\mathrm{I}=\{\mathrm{I}(\mathrm{i}, \mathrm{j})\}$ and membership function values are denoted by $\left\{\mu_{\mathrm{ij}}\right\}$.

Definition of information (H): Information set is denoted by $\mathrm{H}=\left\{\mathrm{H}_{\mathrm{ij}}\right\}$ and is defined as a product of information source values $\left\{\mathrm{I}_{\mathrm{ij}}\right\}$ and corresponding membership function values $\left\{\mu_{\mathrm{ij}}\right\}$ i.e. $\mathrm{H}=\left\{\mathrm{H}_{\mathrm{ij}}\right\}=\left\{\mathrm{I}_{\mathrm{ij}} \mu_{\mathrm{ij}}\right\}$.

The information $(\mathrm{H})$ is defined as:

$$
H=\sum_{i=1}^{n} \sum_{j=1}^{n} H_{i j}
$$

This relation comes from the non-normalized HanmanAnirban entropy function which is defined as:

$$
H=\sum_{i=1}^{n} \sum_{j=1}^{n} p_{i j} e^{-\left(a p_{i j}^{3}+b p_{i j}^{2}+c p_{i j}+d\right)}
$$

Where $\mathrm{p}_{\mathrm{ij}}$ are probabilities such that $\sum_{\mathrm{i}} \sum_{\mathrm{j}} \mathrm{p}_{\mathrm{ij}}=1$, and $\mathrm{a}, \mathrm{b}$, $\mathrm{c}$ and $\mathrm{d}$ are the real- valued parameters that are selected to convert the exponential function into a membership function.

\section{PROOF OF INFORMATION H}

Hanman-Anirban entropy function is defined as:

$$
H=\sum_{i=1}^{n} \sum_{j=1}^{n} p_{i j} e^{-\left(a p_{i j}^{3}+b p_{i j}^{2}+c p_{i j}+d\right)}
$$

Where a, b, c and d are real valued parameters. We will now make use of this entropy function to derive information set.

First take $a=0, b=0, p=I_{i j} ; c=-\frac{1}{\mathrm{f}_{h}^{2}(\text { ref })} ; d=\frac{I_{(\text {ref })}}{\mathrm{f}_{h}^{2}(\text { ref })}$ in Eq. (3) resulting in information $\mathrm{H}$ and is given by

$$
H_{e}=\frac{1}{n^{2}} \sum \sum I_{i j} \mu_{i j}{ }^{e}
$$

Where $\mu_{\mathrm{ij}}^{\mathrm{e}}$ is an exponential membership function and is defined as
$\mu_{\mathrm{ij}}^{\mathrm{e}}=\mathrm{e}^{\frac{-\left|\mathrm{I}_{\mathrm{ij}}-\mathrm{I}_{\mathrm{ref}}\right|}{\mathrm{f}_{\mathrm{h}(\mathrm{ref})}^{2}}}$

The division by $n^{2}$ (n x $\mathrm{n}$ is the size of window) is needed in (5) to make the entropy value within the range $[0,1]$.

Next if we take $p=I_{i j}, a=0, b=\frac{1}{2 f_{h}^{2}(\text { ref })}, c=\frac{-2 \mathrm{I}_{(\text {ref })}}{2 f_{h(\text { ref })}^{2}}, d=$ $\frac{\mathrm{I}_{(\mathrm{ref})}^{2}}{2 \mathrm{f}_{\mathrm{h}(\mathrm{ref})}^{2}}$ eq.(3) becomes another kind of information i.e.

$$
H_{g}=\frac{1}{n^{2}} \sum \sum I_{i j} \mu_{i j}^{g}
$$

Where $\mu_{\mathrm{ij}}^{\mathrm{g}}$ is the Gaussian membership function and is defined as:

$\boldsymbol{\mu}_{i j}^{g}=e^{-\left[\frac{\left|I_{i j}-I_{r e f}\right|}{\sqrt{2 f_{h}}}{ }^{2}\right.}$

And $\mathrm{f}_{\mathrm{h}}^{2}$ is the fuzzifier and is defined as $f_{h}^{2}=\frac{\sum_{i=1}^{W} \sum_{j=1}^{W}\left(I_{r e f}-I_{i j}\right)^{4}}{\sum_{i=1}^{W} \sum_{j=1}^{W}\left(I_{r e f}-I_{i j}\right)^{2}}$

Here $\mathrm{I}_{\text {ref }}$ can be taken as the maximum or average gray level in the window of size $\mathrm{W} \mathrm{X} \mathrm{W.}$

Hence information set $(\mathrm{H})$ can be written as:

$H=\sum_{i=1}^{W} \sum_{j=1}^{W} I_{i j} \mu_{i j}$

Where $\left\{\mathrm{I}_{\mathrm{ij}}\right\}$ is the information source value and $\left\{\mu_{\mathrm{ij}}\right\}$ is the membership function.

Note: With the different value of $\left\{\mu_{\mathrm{ij}}\right\}$ new information $(\mathrm{H})$ can be derived.

\subsection{NEW FEATURES}

Depending upon the information set values some new feature values can be computed which are as follows:

\subsubsection{Energy feature}

The Energy in kth window of size $\mathrm{n} x \mathrm{n}$ is expressed as: 
$E_{k}=\frac{1}{n^{2}} \sum_{i=1}^{n} \sum_{j=1}^{n} \bar{\mu}_{i j} I_{i j}$

Where $n 2=n \quad x \quad$ i.e. the size of window and $\bar{\mu}_{i j}=1-\mu_{i j}$

In this work $\mu_{\mathrm{ij}}=\mathrm{e}^{-\mid \mathrm{I}_{\mathrm{ij}}-\text { mean }\left(\mathrm{I}_{\mathrm{ij}}\right) \mid}$ and is taken according to the experimentation in which energy value is found to give the best performance for the system.

\subsubsection{Sigmoid feature}

The sigmoid feature in kth window of size $\mathrm{n} X$ is expressed as

$$
S_{g}^{k}=\frac{1}{n^{2}} \sum_{i=1}^{n} \sum_{j=1}^{n} \frac{I_{a v g}}{1+e^{-\mu_{i j} I_{i j}}}
$$

Where $I_{\text {avg }}$ is the average number of gray level values and is expressed as:

$$
\mathrm{I}_{\mathrm{avg}}=\frac{\sum_{\mathrm{i}=1}^{\mathrm{m}} \sum_{\mathrm{j}=1}^{\mathrm{n}} \mathrm{I}_{\mathrm{ij}}}{\mathrm{n}^{2}}
$$

Here $\mu_{\mathrm{ij}}=\mathrm{e}^{-\mid \mathrm{I}_{\mathrm{ij}}-\text { mean }\left(\mathrm{I}_{\mathrm{ij}}\right) \mid}$ and is taken according to the experimentation in which sigmoid value is found to give the best performance for the system.

\subsection{Algorithm For Energy And Sigmoid Feature Extraction}

1. Divide the Region of interest (ROI) image into non overlapping windows of size WXW.

2. Extract the local information from each window by computing energy and sigmoid feature. The local information can be changed by changing the choice of membership function.

3. Repeat step 2 for all the windows of size $7 X 7$, 9X9, 11X11, 13X13, and $17 \mathrm{X} 17$ to compute the performance.

4. Concatenate the resulted features from all the windows to form a feature vector.

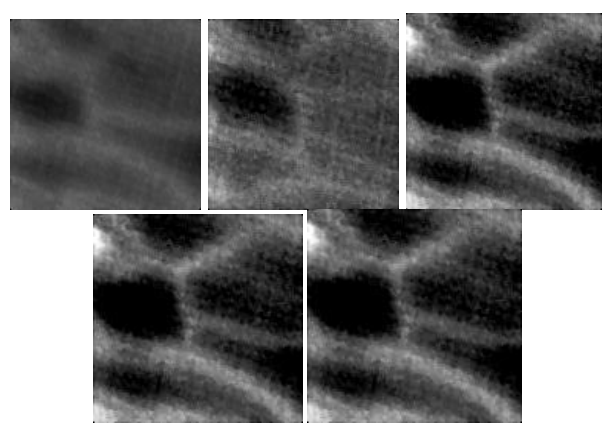

Figure2: ROI Sample Images of one User

\section{HANMAN CLASSIFIER}

Hanman classifier is used for classification of extracted features. This classifier is based on T-norms and is expressed as

$$
\begin{gathered}
H_{m h}(i)=\sum_{j} E_{m h}^{i}(j) e^{-E_{m h}^{i}(j)}, m \neq h, i \\
=1,2, \ldots, c
\end{gathered}
$$

\subsection{Triangular Norms}

T-norms are the generalization of minimum operator. These are the binary operator mainly used in probabilistic metric spaces and multi-valued logics. These norms are used in deriving the Hanman classifier (described in the section 4.2). These norms are applied in various fields such as fuzzy set theory, fuzzy logic and generalized measures.

Definition: T-norms is a function that maps unit square into unit function and is denoted by $\mathrm{T}(\mathrm{a}, \mathrm{b})$. This function is expressed asT: $[0,1] \mathrm{X}[0,1] \rightarrow[0,1]$. This function satisfies the following properties:

$$
\begin{aligned}
& \text { 1. Commutativity: } T(a, b)=T(b, a) \\
& \text { 2. Monotonicity: } T(a, b) \leq T(c, d) \text {, if } a \leq \\
& c \text {, and } b \leq d \\
& \text { 3. Associativity: } T(a, T(b, c))=T(T(a, b), c) \\
& \text { 4. Identity: The number } 1 \text { is a neutral element. }
\end{aligned}
$$

\subsection{Hanman Classifier Based On T-Norms}

Let the size of the extracted feature vector be n. Now divide the feature vector into training samples and testing samples. Let the number of training samples are $T_{r}$ and the testing samples are $\left(\mathrm{T}_{\mathrm{S}_{1}}, \mathrm{~T}_{\mathrm{S}_{2}} \ldots \ldots \ldots . \mathrm{T}_{\mathrm{S}_{\mathrm{n}}}\right)$.

First normalize the feature vector using Min-Max normalization. Let the resulted normalized matrix of $i^{\text {th }}$ user be denoted by $\mathrm{X}^{\mathrm{i}}$ and is represented as

$$
X^{i}=\left(\begin{array}{cccc}
x_{11}^{i} & x_{12}^{i} & \cdots & x_{1 n}^{i} \\
\vdots & \vdots & \ddots & \vdots \\
x_{T_{r_{1}}}^{i} & x_{T_{r_{2}}}^{i} & \cdots & x_{T_{r_{n}}}^{i}
\end{array}\right)
$$

Where, each row of the matrix represents a training sample of the $\mathrm{i}^{\text {th }}$ user. Now compute the error vector $\left(\mathrm{e}_{\mathrm{mj}}^{\mathrm{i}}\right)$ between training samples and testing samples of $\mathrm{i}^{\text {th }}$ user which is defined as:

$$
\begin{aligned}
\mathrm{e}_{\mathrm{mj}}^{\mathrm{i}} & =\left|\mathrm{x}_{\mathrm{mj}}^{\mathrm{i}}-\mathrm{t}_{\mathrm{s}_{\mathrm{j}}}\right| \text { where } \mathrm{m}=1,2, \ldots \ldots, \mathrm{s} \text { and } \mathrm{j}= \\
1,2, \ldots \ldots \ldots, \mathrm{n}(7) &
\end{aligned}
$$

Where, $x_{m j}^{i}$ denotes the $m^{\text {th }}$ training sample

Now if the test sample belongs to $\mathrm{i}^{\text {th }}$ user then the errors computed from eq. (7) will be close to 0 .

Note that $\mathrm{T}$-norms of the errors $\mathrm{e}_{\mathrm{mj}}^{\mathrm{i}}$ (error in the $\mathrm{j}^{\text {th }}$ feature between the $m^{\text {th }}$ training sample and the test sample) and $e_{h j}^{i}$ (error in the $\mathrm{j}^{\text {th }}$ feature between the $\mathrm{h}^{\text {th }}$ training sample and the test sample) in $\mathrm{j}^{\text {th }}$ feature gives the infinite number of the errors depending on the T-norm used. If the test sample belongs to the $\mathrm{i}^{\text {th }}$ user then both $\mathrm{e}_{\mathrm{mj}}^{\mathrm{i}}$ and $\mathrm{e}_{\mathrm{hj}}^{\mathrm{i}}$ will be small Hence we can say that $\mathrm{T}\left(\mathrm{e}_{\mathrm{mj}}^{\mathrm{l}}, \mathrm{e}_{\mathrm{hj}}^{\mathrm{l}}\right)<\min \left(\mathrm{e}_{\mathrm{mj}}^{\mathrm{l}}, \mathrm{e}_{\mathrm{hj}}^{\mathrm{l}}\right)$.

Now compute T-norm error vector which is given by:

$$
E_{m h}^{i}(j)=T\left(e_{m j}^{i}, e_{h j}^{i}\right), m \neq h
$$

Note: The vector $E_{m h}^{i}(j)$ can also be used for classification but the entropy of this vector is inequitable.

Now recall Hanman-Anirban entropy function which is defined as: 


$$
H=\sum_{i=1}^{n} \sum_{j=1}^{n} p_{i j} e^{-\left(a p_{i j}^{3}+b p_{i j}^{2}+c p_{i j}+d\right)}
$$

Here by taking $a=0, b=0, p_{j}=T\left(e_{m j}^{i}, e_{h j}^{i}\right)=E_{m h}^{i}(j)$ the T-normed entropy becomes $\mathrm{H}_{\mathrm{mh}}(\mathrm{i})$ and is defined as:

$$
H_{m h}(i)=\sum_{j} E_{m h}^{i}(j) e^{-\left(c E_{m h}^{i}(j)+d\right)}, m \neq h
$$

Where $\mathrm{c}$ and $\mathrm{d}$ are the learning parameters and $\mathrm{m}, \mathrm{h}=$ $1,2, \ldots . ., \mathrm{T}_{\mathrm{r}}, \mathrm{i}=1,2, \ldots, \mathrm{c}$

To avoid learning we take $\mathrm{c}=1, \mathrm{~d}=0$ in Eq. (9). This leads to the Hanman classier i.e.

$$
H_{m h}(i)=\sum_{j} E_{m h}^{i}(j) e^{-E_{m h}^{i}(j)}, m \neq h, i=1,2, \ldots, c
$$

The choice of T-norms is essential for the success of the classier. In our work, we have used Frank T-norm which is found to be more appropriate and is given by

$$
\mathrm{T}(\mathrm{x}, \mathrm{y})=\log _{\mathrm{p}}\left(1+\frac{\left(\mathrm{p}^{\mathrm{x}}-1\right)\left(\mathrm{p}^{\mathrm{y}}-1\right)}{\mathrm{p}-1}\right)
$$

Where $\mathrm{p}$ is the unknown parameter

\subsection{Algorithm For Hanman Classifier}

1. Divide the dataset into training $\operatorname{samples}\left(T_{r}\right)$ and testing samples $\left(T_{S}\right)$.

2. Normalize the feature vector using Min-Max normalization.

3. Now compute absolute error vector between training samples and testing samples. Resulted error vector is given by: $\left(e_{m 1}^{i}, e_{m 2}^{i}, \ldots . ., e_{m n}^{i}\right)$ Where $\mathrm{m}=1,2, \ldots, T_{r}$

4. Compute T-normed error vector: It is computed by comparing the pair of error vector $\left(e_{m 1}^{i}, e_{m 2}^{i}, \ldots . ., e_{m n}^{i}\right) \operatorname{and}\left(e_{h 1}^{i}, e_{h 2}^{i}, \ldots . ., e_{h n}^{i}\right)$ and is denoted by $E_{m h}^{i}(j)$ (where $\left.\mathrm{j}=1,2, \ldots, \mathrm{n}\right)$ for $i^{\text {th }}$ user.

5. Evaluate $H_{m h}(i) \forall m, h=1,2, \ldots, T_{r}$ and the compute $G_{i}=\sum_{m \neq h=1}^{T_{r}} H_{m h}(i)$.

6. Repeat step 2-4for all users $(i=1,2, \ldots \ldots, C)$ and if $k=i n f_{i}\{i\}$, then the test user gets identified with $k^{\text {th }}$ user i.e. $G_{k}<G_{i} \forall i \neq k$.

\subsection{Optimization Of Hanman Classifier Performance}

We have optimized the performance of Hanman classifier by using genetic algorithm. Genetic algorithms are used to optimize non linear systems with large number of variables. In this we are concerned with the optimization of the unknown parameter ' $p$ ' present in accuracy function. By taking different values of ' $p$ ' we have optimize hanman classifier to get the best value of accuracy.

\section{EXPERIMENTAL RESULTS}

This section is divided into three steps i.e. feature extraction, classification and verification.

\subsection{Feature Extraction}

Feature extraction has been done on ROI images of hand vein database developed at IIT, Delhi. For feature extraction the images are partitioned into non overlapping windows of size $\mathrm{n} \mathrm{X} \mathrm{n}$. Then two new local features i.e. energy feature and sigmoid feature are extracted from each window. These features have been extracted for window size of 7X7, 9X9, 11X11, 13X13, and 17X17.

\subsection{Classification}

After feature extraction the classification has been done on extracted features. In classification the performance of all the extracted features is evaluated by using Hanman classifier in terms of accuracy. Hanman classifier generates the genuine score and imposter scores. In this work for each instance of hand vein datasets there are 100 users and each user has 5 images. We have taken 4 images as training samples and 1 image as testing samples. We have also performed cross validation keeping 1 image as test and rest of the images as train and we are continuously changing the test images by next image of the user keeping rest of the images as train images to compute the performance. Genuine scores are obtained by comparing the feature vectors of images of the same user. Impostor scores are obtained by comparing the feature vectors of images of different users. In our work there are $100 * 4$ genuine scores and 500*499*4 imposter scores. After classification we have optimized the hanman classifier so as to improve the performance of classifier. The results are shown below

Table 1: comparison of accuracy of different classifiers

\begin{tabular}{|l|l|l|l|l|l|l|}
\hline $\begin{array}{l}\text { Window } \\
\text { Size }\end{array}$ & \multicolumn{2}{|l|}{ Hanman classifier } & \multicolumn{2}{|l|}{$\begin{array}{l}\text { k-nearest } \\
\text { neighbor } \\
\text { classifier }\end{array}$} & \multicolumn{2}{|c|}{ SvM } \\
\hline & Energy & Sigmoid & Energy & Sigmoid & Energy & Sigmoid \\
\hline $7 X 7$ & 97 & 98 & 96 & 98 & 97 & 98 \\
\hline $9 X 9$ & 96 & 98 & 96 & 98 & 96 & 97 \\
\hline $11 X 11$ & 96 & 97 & 96 & 97 & 95 & 97 \\
\hline $13 X 13$ & 97 & 98 & 96.5 & 98 & 96 & 97 \\
\hline $17 X 17$ & 95 & 97 & 94.5 & 97 & 94 & 96 \\
\hline
\end{tabular}

Table 2: Accuracy obtained by hanman classifier before optimization

\begin{tabular}{|l|l|l|}
\hline Window size & $\begin{array}{l}\text { Energy } \\
\text { feature }\end{array}$ & $\begin{array}{l}\text { Sigmoid } \\
\text { feature }\end{array}$ \\
\hline 7 X7 & 97 & 98 \\
\hline $9 \times 9$ & 96 & 98 \\
\hline 11 X11 & 96 & 97 \\
\hline $13 \times 13$ & 97 & 98 \\
\hline $17 \times 17$ & 95 & 97 \\
\hline
\end{tabular}


Table 3: Accuracy obtained by hanman classifier after optimization

\begin{tabular}{|l|l|l|}
\hline Window size & $\begin{array}{l}\text { Energy } \\
\text { feature }\end{array}$ & $\begin{array}{l}\text { Sigmoid } \\
\text { feature }\end{array}$ \\
\hline 7 X7 & 98 & 99 \\
\hline 9 X9 & 97 & 99 \\
\hline 11 X11 & 97 & 98 \\
\hline 13 X13 & 98 & 99 \\
\hline $17 X 17$ & 97 & 98 \\
\hline
\end{tabular}

\subsection{Verification}

After classification, verification has been done. In verification the computed genuine scores and imposter scores are compared with the threshold value to calculate the error rates i.e. the FAR (false acceptance rate) and FRR (false rejection rate) are computed.

Table 4: FAR and FRR for information sets based features

\begin{tabular}{|c|c|c|}
\hline No of images & FAR (\%) & FRR (\%) \\
\hline 10 & 0.0001 & 0.0010 \\
\hline 20 & 0.0025 & 0.0050 \\
\hline 30 & 0.0060 & 0.0080 \\
\hline 40 & 0.0080 & 0.0100 \\
\hline 50 & 0.0090 & 0.0125 \\
\hline 60 & 0.0105 & 00140 \\
\hline 70 & 0.0125 & 0.0170 \\
\hline 80 & 0.0140 & 0.0190 \\
\hline 90 & 0.0160 & 0.0200 \\
\hline 100 & 0.0200 & 0.0300 \\
\hline
\end{tabular}

The initial value of the threshold is set to the minimum of the genuine scores and the final value of the threshold becomes the maximum of impostor scores. The Receiver Operating Characteristics (ROC) curve which is a plot of GAR vs FAR is obtained by computing the values of (FAR, FRR) for varying thresholds. Best values of energy feature and sigmoid feature by hanman classifier in both the cases i.e. before optimization and after optimization are coming out for window size $7 \mathrm{X} 7$ and $13 \mathrm{X} 13$.

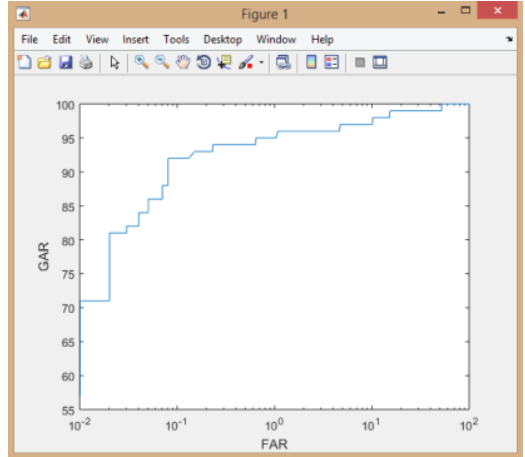

a. window size $7 \mathrm{X} 7$

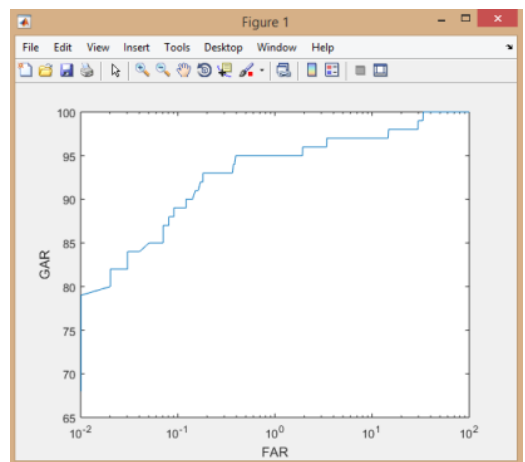

b. window size $13 \times 13$

Figure 3: a. GAR and FAR graph for energy feature with window size 7X7 b. GAR and FAR graph for energy feature with window size $13 \times 13$

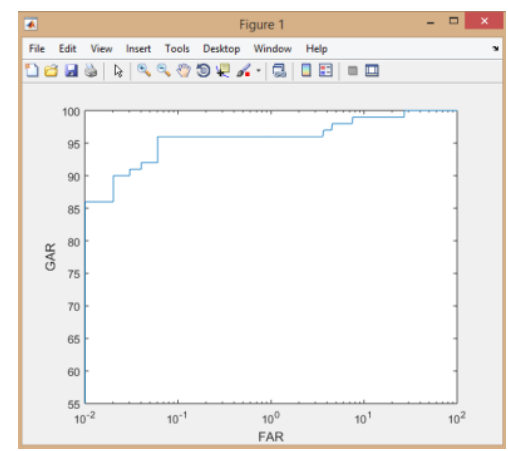

c. window size $7 \mathrm{X} 7$

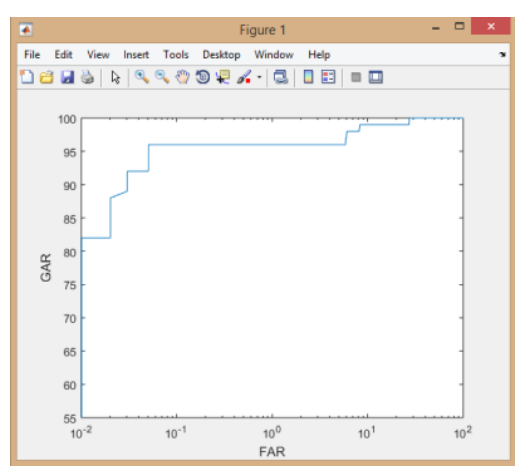

d. window size $13 \times 13$

Figure 4: a. GAR and FAR graph for sigmoid feature with window size 7X7 b. GAR and FAR graph for sigmoid feature with window size $13 X 13$ 


\section{CONCLUSION}

In this paper information sets concept has been used for feature extraction of hand veins. Two types of features are extracted i.e. energy feature and sigmoid feature. According to the accuracy calculated by Hanman classifier sigmoid features are found to be more efficient and reliable. Then a comparison been done to compare the accuracy obtained from hanman classifier with accuracy computed from two different classifiers i.e. k-nearest neighbor classifier and SVM classifier. Then optimization of hanman classifier is done so as to improve the performance. The accuracy obtained after optimization shows that that the proposed system is $99 \%$ efficient.

\section{REFERENCES}

[1] L.Chen, H.Zheng, L.Li , P.Xie and S.Lui, “ Nearinfrared Dorsal Hand Vein Image Segmentation by Local Thresholding Using Grayscale Morphology", The 1st International Conference on Bioinformatics and Biomedical Engineering, 2007. Page(s):868 - 871.

[2] Tanaka, T. \& Kubo, K.," Biometric authentication by hand vein patterns", In the Proceedings of SICE Annual Conference. Yokohama, Japan, 2004.

[3] Wang, L., Leedham, G. and Cho, D. S., "Infrared imaging of hand vein patterns for biometric purposes", IET Comput. Vis., 2007, 1, 113-122.

[4] Shahin, M.; Badawi, A. \& Kamel, M.," Biometric authentication using fast correlation of near infrared hand vein patterns", Int. J. Biomed. Sci., 2007, 2(3), 141-148.

[5] Kumar, A, and Prathyusha, K. V," Personal Authentication Using Hand Vein Triangulation and Knuckle Shape", IEEE Transactions on Image processing, Vol. 18, No. 9, 2009, pp. 2127-2136

[6] Wang, Y.; Wang, D.; Liu, T. \& Li, X., "Local SIFT analysis for hand vein pattern verification", In the
Proceedings of the SPIE International Conference on Optical Instruments and Technology: Optoelectronic Information Security Shanghai, China, 2009.

[7] Wang, Y., Kefeng, L., Cui, J., Shark, L. K. and Varley, M. "Study of hand-dorsa vein recognition", Proc. 6th Int. Conf. on Intelligent computing: ICIC '10, Changsha, China, August 2010, Hunan University, Vol. 1, pp. 490-498.

[8] Wang, Y.; Li, K. \& Cui, J.," Hand-dorsa vein recognition based on partition local binary pattern" ,In the Proceedings of 10th IEEE International Conference on Signal Processing. Beijing, China, 2010.

[9] Wang, Y. \& Liao, W. ,“ Hand vein recognition based on feature coding", In the Proceedings of the 7th Chinese conference on Biometric Recognition. Guangzhou, China, 2012.

[10] Tang, Y.; Huang, D. \& Wang, Y., "Hand-dorsa vein recognition based on multi-level key point detection and local feature matching", In the Proceedings of the 21st International Conference on Pattern Recognition. Tsukuba, Japan, 2012.

[11] Huang, D.; Tang, Y.; Wang, Y. \& Chen, L..”Hand vein recognition based on oriented gradient maps and local feature matching", In the Proceedings of the 11th Asian Conference on Computer Vision. Verlag, Berlin Heidelberg, 2012.

[12] Mamta, Madasu Hanmandlu ," Robust ear based authentication using Local Principal Independent Components," Expert Systems with Applications 40 (2013) 6478-6490, pg 24. 